\title{
SIRT1 is required for mitochondrial biogenesis reprogramming in hypoxic human pulmonary arteriolar smooth muscle cells
}

\author{
PENGYUN LI ${ }^{1,2}$, YAN LIU ${ }^{3}$, NANA BURNS ${ }^{4}$, KE-SENG ZHAO ${ }^{1}$ and RUI SONG ${ }^{1}$ \\ ${ }^{1}$ Guangdong Key Laboratory of Shock and Microcirculation Research, Department of Pathophysiology, \\ Southern Medical University, Guangzhou, Guangdong 510515; ${ }^{2}$ Key Laboratory of Medical Electrophysiology, \\ and Institute of Cardiovascular Research, Southwest Medical University, Luzhou, Sichuan 646000; \\ ${ }^{3}$ Department of Pharmacy, Henan Medical College, Zhengzhou, Henan 450046; \\ ${ }^{4}$ Department of Pediatrics, University of Colorado Denver, Aurora, CO 80045, USA
}

Received October 13, 2016; Accepted March 16, 2017

DOI: 10.3892/ijmm.2017.2932

\begin{abstract}
Although recent studies have reported that mitochondria are putative oxygen sensors underlying hypoxic pulmonary vasoconstriction, little is known concerning the sirtuin 1 (SIRT1)-mediated mitochondrial biogenesis regulatory program in pulmonary arteriolar smooth muscle cells (PASMCs) during hypoxia/reoxygenation (H/R). We investigated the epigenetic regulatory mechanism of mitochondrial biogenesis and function in human PASMCs during $\mathrm{H} / \mathrm{R}$. Human PASMCs were exposed to hypoxia of 24-48 $\mathrm{h}$ and reoxygenation of 24-48 $\mathrm{h}$. The expression of SIRT1 was reduced in a time-dependent manner. Mitochondrial transcription factor A (TFAM) expression was increased during hypoxia and decreased during reoxygenation, while the release of TFAM was increased in a time-dependent manner. Lentiviral overexpression of SIRT1 preserved SIRT3 deacetylase activity in human PASMCs exposed to H/R. Knockdown of PGC-1 $\alpha$ suppressed the effect of SIRT1 on SIRT3 activity. Knockdown of SIRT3 abrogated SIRT1-mediated deacetylation of cyclophilin D (CyPD). Notably, knockdown of SIRT3 or PGC-1 $\alpha$ suppressed the incremental effect of SIRT1 on mitochondrial TFAM, mitochondrial DNA (mtDNA) content and cellular ATP levels. Importantly, polydatin restored SIRT1 levels in human PASMCs exposed to H/R. Knockdown of SIRT1 suppressed the effect of polydatin on mitochondrial TFAM, mtDNA content and cellular ATP levels. In conclusion, SIRT1 expression is decreased in human PASMCs during $H / R$. TFAM expression in mitochondria is reduced and the
\end{abstract}

Correspondence to: Dr Rui Song or Dr Ke-Seng Zhao, Guangdong Key Laboratory of Shock and Microcirculation Research, Department of Pathophysiology, Southern Medical University, Guangzhou, Guangdong 510515, P.R. China

E-mail: songrui110@126.com

E-mail: zhaoks@fimmu.com

Key words: sirtuin, pulmonary arteriolar smooth muscle cells, mitochondria, hypoxia, polydatin release of TFAM is increased by H/R. PGC-1 $\alpha /$ SIRT3/CyPD mediates the protective effect of SIRT1 on expression and release of TFAM and mitochondrial biogenesis and function. Polydatin improves mitochondrial biogenesis and function by enhancing SIRT1 expression in hypoxic human PASMCs.

\section{Introduction}

Pulmonary arteriolar smooth muscle cells (PASMCs) play an important role in pulmonary arterial remodeling and hypoxic pulmonary vasoconstriction during sustained hypoxia or ischemia and reperfusion. A limited understanding of the basic cellular mechanisms governing this disease process has led to few therapeutic options and continued morbidity and mortality $(1,2)$.

In the pulmonary vasculature, mitochondria contribute to physiological intracellular signaling pathways through production of reactive oxygen species and play the role of oxygen sensors that coordinate hypoxic pulmonary vasoconstriction $(3,4)$. Mitochondrial alterations have been observed in pulmonary arteries in pulmonary hypertension and mitochondrial dysfunction plays a role in the pathogenesis of pulmonary hypertension (4). Several intra- and extra-mitochondrial causes of mitochondrial dysfunction in pulmonary hypertension have been described in pulmonary arteries, whereas ischemia and decreased angiogenesis appear to be the main triggers of mitochondrial dysfunction (5). Mitochondrial-targeted therapies are currently being investigated and may open novel therapeutic perspectives in hypoxic pulmonary vasoconstriction and pulmonary hypertension. Decreased oxidative capacity and energy production are due to decreased mitochondrial biogenesis. It is unknown what mechanism of mitochondrial biogenesis reprogramming in PASMCs occurs during hypoxia/ reoxygenation $(\mathrm{H} / \mathrm{R})$.

Recently, histone deacetylase sirtuin 1 (SIRT1) has emerged as a crucial regulator of mitochondrial function in vascular smooth muscle, liver, kidney and heart (6-9). SIRT1 and SIRT3 also play roles in pulmonary hypertension $(10,11)$. The effects of SIRT1 on mitochondrial biogenesis are mediated by PGC-1 $\alpha$ (12). Knockdown of Sirt3 blocks PGC-1 $\alpha$-dependent gene expression of subunits I and II 
(mitochondrial encoded) as well as subunit VIIa (nuclear encoded) from cytochrome $c$ oxidase, respectively (13). SIRT3 is required for PGC-1 $\alpha$-induced mitochondrial-related gene expression and mitochondrial biogenesis. SIRT1 and SIRT3 have been reported to play an important role in mitochondrial morphology and function in aorterial smooth muscle cells (6). Cyclophilin D (CyPD), a peptidylprolyl isomerase F (PPIase), modulates mitochondrial membrane permeability (14). SIRT1/SIRT3 mediates deacetylation of CyPD to regulate mitochondrial membrane permeability and mitochondrial function during hemorrhagic shock (6). However, both SIRT1 and SIRT3, in regard to mitochondrial biogenesis, are still lacking in PASMCs during $\mathrm{H} / \mathrm{R}$.

Mitochondrial transcription factor A (TFAM) is a multi-functional DNA binding protein that is essential for transcriptional activation and mitochondrial DNA (mtDNA) organization. TFAM is an important mitochondrial biogenesis marker (15). In a clinical trial, caloric restriction improved skeletal muscle mitochondrial function and mitochondrial biogenesis through regulation of SIRT1, PGC- $1 \alpha$ and TFAM gene expression (16). TFAM can also act as one of the mitochondrial damage-associated molecular patterns (DAMPs), which may be associated with vascular damage (17).

Polydatin is a glucoside of resveratrol, a natural polyphenolic compound. Polydatin protects multiple organs from ischemia/reperfusion injury (18-20). A previous study from our laboratory showed that polydatin protects against ASMC mitochondrial injury in hemorrhagic shock (21). Recently, we found that polydatin protects mitochondria through activation of SIRT1 in the liver and small intestine during hemorrhagic shock $(20,22)$. Yet, the role of SIRT1 in mitochondrial biogenesis during hypoxia and reperfusion remains unclear.

We hypothesized that SIRT1 may play an important role in protection against mitochondrial biogenesis reprogramming and function in PASMCs during H/R. Polydatin protects mitochondrial biogenesis through SIRT1. The purpose of this study was to determine: i) the expression and activity of SIRT1 in PASMCs during $\mathrm{H} / \mathrm{R}$, ii) the expression of TFAM in mitochondria and the release of TFAM during H/R, iii) the role of PGC-1 $\alpha /$ SIRT3/CyPD in the effect of SIRT1 on mitochondrial biogenesis during $\mathrm{H} / \mathrm{R}$, and iv) the effect of polydatin on mitochondrial biogenesis.

\section{Materials and methods}

Materials. Antibodies against human SIRT1 (\#2310), SIRT3 (\#2627), PGC-1 $\alpha$ (\#2178) and acetylated-lysine (\#9441) were all purchased from Cell Signaling Technology, Inc. (Beverly, MA,USA). The antibody against human CyPD (\#ab110324) and mtTFA Human SimpleStep ELISA ${ }^{\mathrm{TM}}$ kit were purchased from Abcam, Inc. (Cambridge, MA, USA). The SIRT3 Deacetylase Fluorometric assay kit was purchased from CycLex Co., Ltd. (Nagano, Japan). SIRT1 open reading frame (ORF) and control vector were both obtained from the Functional Genomics Facility of the University of Colorado. Lipofectamine 2000 was purchased from Life Technologies, Inc. (Grand Island, NY, USA). TransDux ${ }^{\mathrm{TM}}$ transduction reagent was purchased from System Biosciences, Inc. (Mountain View, CA, USA). Human Mitochondrial to Nuclear DNA Ratio kit was purchased from Clontech Laboratories, Inc. (Mountain View,
CA, USA). CellTiter-Glo Luciferase-based assay was purchased from Promega (Madison, WI, USA). Specific siRNA for human SIRT3 and PGC-1 $\alpha$ and scrambled siRNA were purchased from Integrated DNA Technologies, Inc. (Coralville, IA, USA). HiPerFect ${ }^{\circledR}$ transfection reagent and other transfection-related reagents were purchased from Qiagen (Valencia, CA, USA). Dulbecco's modified Eagle's medium (DMEM) was purchased from Life Technologies. All other chemicals and reagents were from Sigma-Aldrich Chemical Co. (St. Louis, MO, USA).

Cell preparation and culture. Human PASMCs were cultured by explant outgrowth from the branches between an artery and a bronchiole harvested for lung transplantation. Cells were grown in DMEM supplemented with $10 \%$ (vol/vol) fetal bovine serum (FBS), $100 \mathrm{U} / \mathrm{ml}$ penicillin, $100 \mu \mathrm{g} / \mathrm{ml}$ streptomycin and $1.25 \mu \mathrm{g} / \mathrm{ml}$ amphotericin $\mathrm{B}$. The cells exhibited the typical 'hill and valley' growth morphology of SMCs. PASMC isolates lacked endothelial cells and fibroblasts as verified by $\alpha$-SMA, fibroblast-specific protein 1 (FSP1) and von Willebrand factor (vWF) staining. Cells from passages 3-6 were used for the experiments.

Mitochondrial DNA content. Genomic DNA (including mtDNA) was extracted from human PASMCs. Mitochondrial DNA content was measured using the human mitochondrial to Nuclear DNA Ratio kit according to the manufacturer's instructions. The copy number of mtDNA was calculated as the average of the values and calculated based on the combinations of the primers. Data analysis was performed and the mtDNA copy number was determined by relative quantification.

Mitochondrial TFAM levels and TFAM release. Mitochondrial TFAM levels and TFAM release were measured with the enzyme-linked immunosorbent assay TFAM SimpleStep. To measure mitochondrial TFAM levels, the mitochondria in the PASMCs were isolated. To measure the release of TFAM, cell culture supernatants were collected. Particulates were removed by centrifugation for $15 \mathrm{~min}$ at $10,000 \mathrm{x} \mathrm{g}$ at $2-8^{\circ} \mathrm{C}$. Then mitochondrial TFAM levels and TFAM release were assessed according to the assay procedure of the TFAM SimpleStep ELISA kit. Measurements were carried out in quadruplicates.

Measurement of intracellular ATP. PASMC ATP levels were measured by a luciferase-based assay (CellTiter-Glo; Promega) according to the manufacturer's protocols. The luminescence was recorded using an automatic microplate reader (SpectraMax M5; Molecular Devices, Sunnyvale, CA, USA).

Western blot analysis. Total protein was isolated from the PASMCs by homogenization in cold RIPA lysis buffer containing protease inhibitors and phosphatase inhibitors (Roche, Indianapolis, IN, USA). Equal amounts of proteins were separated by sodium dodecyl sulfate-polyacrylamide gel electrophoresis (SDS-PAGE) and transferred to a polyvinylidene difluoride membrane. After blocking in 5\% bovine serum albumin (BSA) in Tris-buffered saline/Tween-20 (50 mM Tris, $\mathrm{pH} 7.5,500 \mathrm{mM}$ sodium chloride, and $0.05 \%$ Tween-20) for $1 \mathrm{~h}$ at room temperature, the membranes were incubated overnight 

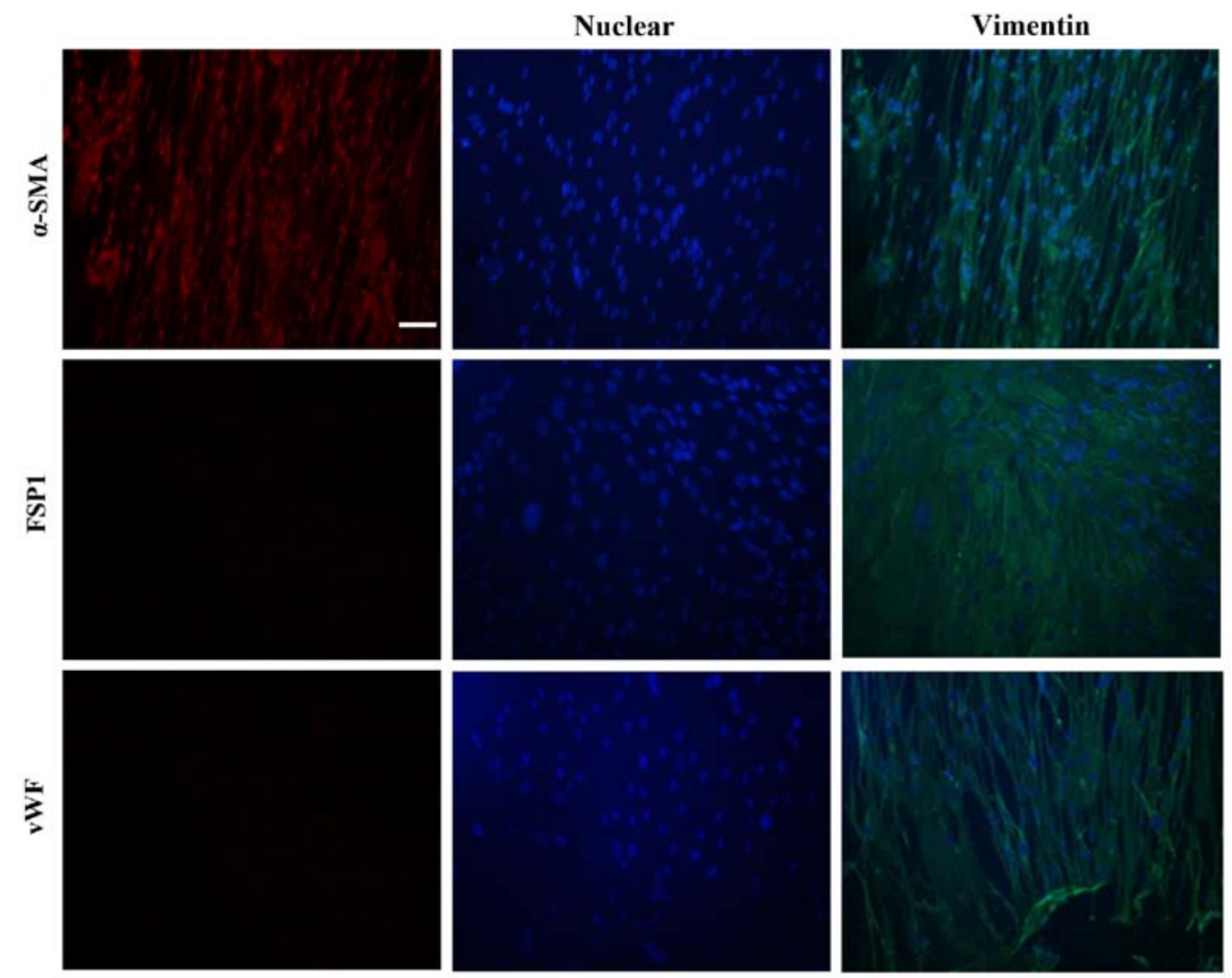

Figure 1. Characterization of isolated human pulmonary arteriolar smooth muscle cells (PASMCs). Immunofluorescent staining was applied to detect $\alpha$-smooth muscle actin ( $\alpha$-SMA), fibroblast-specific protein 1 (FSP1) and von Willebrand factor (vWF). The isolated cells were determined to be smooth muscle cells This was confirmed by double immunofluorescent staining that identified both vimentin and $\alpha$-SMA in these cells. Almost all cells were immunoreactive to the antibody against vimentin (green), and cells were positively labeled by the antibody against $\alpha$-SMA (red) but negatively labeled by the antibodies against FSP1 and vWF. Scale bar, $100 \mu \mathrm{m}$. Blue, nucleus.

at $4^{\circ} \mathrm{C}$ with primary antibodies against SIRT1, SIRT3, PGC- $1 \alpha$, CyPD and $\beta$-actin. After incubation with a secondary antibody conjugated to horseradish peroxidase for $1 \mathrm{~h}$ at room temperature, immunoreactive signals were detected by chemiluminescence using ECL detection reagent, visualized using a chemiluminescence detection system (Image Station 4000R; Kodak, Rochester, NY, USA), and analyzed using Quantity One software (version 4.52).

SIRT3 deacetylase activity assay. For this assay the SIRT3 Deacetylase Fluorometric assay kit was used. Briefly, PASMCs were homogenized in $500 \mu 1$ of immunoprecipitation buffer (T-PER ${ }^{\circledR}$ Tissue Protein Extraction Reagent 78510; Pierce, Rockford, IL, USA). After immunoprecipitation of SIRT3, the final reaction mixtures $(50 \mu \mathrm{l})$ contained $50 \mathrm{mM}$ Tris-HCL (pH 8.8), $4 \mathrm{mM} \mathrm{MgCl}{ }_{2}, 0.5 \mathrm{mM}$ DTT, $0.25 \mathrm{~mA} / \mathrm{ml}$ lysyl endopeptidase, $1 \mu \mathrm{M}$ Trichostatin A, $200 \mu \mathrm{M} \mathrm{NAD}^{+}$ and $5 \mu \mathrm{l}$ of extract. The fluorescence intensity (Ex $340 \mathrm{~nm}$, Em $460 \mathrm{~nm}$ ) was measured using an automatic microplate reader (SpectraMax M5; Molecular Devices, Sunnyvale, CA, USA).

$H / R$. Human PASMCs were cultured in a hypoxic incubator (3\% oxygen, $5 \%$ carbon and $92 \%$ dioxide) for $24-48 \mathrm{~h}$ and then cultured in a normal incubator ( $95 \%$ air and $5 \% \mathrm{CO}_{2}$ ) for $24-48 \mathrm{~h}$.

Gene knockdown and overexpression. To knockdown SIRT3 and PGC-1 $\alpha$, human PASMCs (60-70\% confluent) in 24-well plates were incubated with a mixture of siRNA $(60 \mathrm{nM})$ (Life Technologies) and transfection reagent (Qiagen) for $48 \mathrm{~h}$ (35). Control cells were treated with scrambled siRNA and transfection reagent.

SIRT1 expression plasmids, control plasmid and lentiviral packaging plasmids (pVSVG, pRSV-Rev and pMDL) (GE Dharmacon, Lafayette, CO, USA) were purified using the HiSpeed Plasmid Midi kit (Qiagen). Lentiviruses that overexpress SIRT1 and scrambled control were generated by co-transfection of 293T cells using Lipofectamine 2000 . After $48 \mathrm{~h}$, lentiviral supernatants were collected and concentrated. Human PASMCs were infected with a lentivirus overexpressing SIRT1 or scrambled control using TransDux transduction reagent. The expression of GFP was examined under a fluorescence microscope.

Co-immunoprecipitation. Human PASMCs $(80-90 \%$ confluent) in 6-well plates were gently rinsed 3 times with phosphate-buffered saline (PBS). Cells were lysed in Pierce cell lysis buffer $(25 \mathrm{mM}$ Tris- $\mathrm{HCl}, 150 \mathrm{mM} \mathrm{NaCl}, 1 \mathrm{mM}$ EDTA, $1 \%$ NP-40 and 5\% glycerol, pH 7.4), and the lysates were centrifuged at $13,000 \mathrm{xg}$ for $10 \mathrm{~min}$ at $4^{\circ} \mathrm{C}$. The cleared lysates were incubated with anti-acetylated-lysine antibody (Cell Signaling Technology, Boston, MA, USA) overnight at $4^{\circ} \mathrm{C}$ with rocking. Next, protein A/G-agarose beads (20 $\mu \mathrm{l}$ of $50 \%$ bead slurry) were added and the mixtures were incubated with gentle rocking for $3 \mathrm{~h}$ at $4^{\circ} \mathrm{C}$. The immunoprecipitates were washed 3 times with $0.5 \mathrm{ml}$ of lysis buffer and resuspended in 

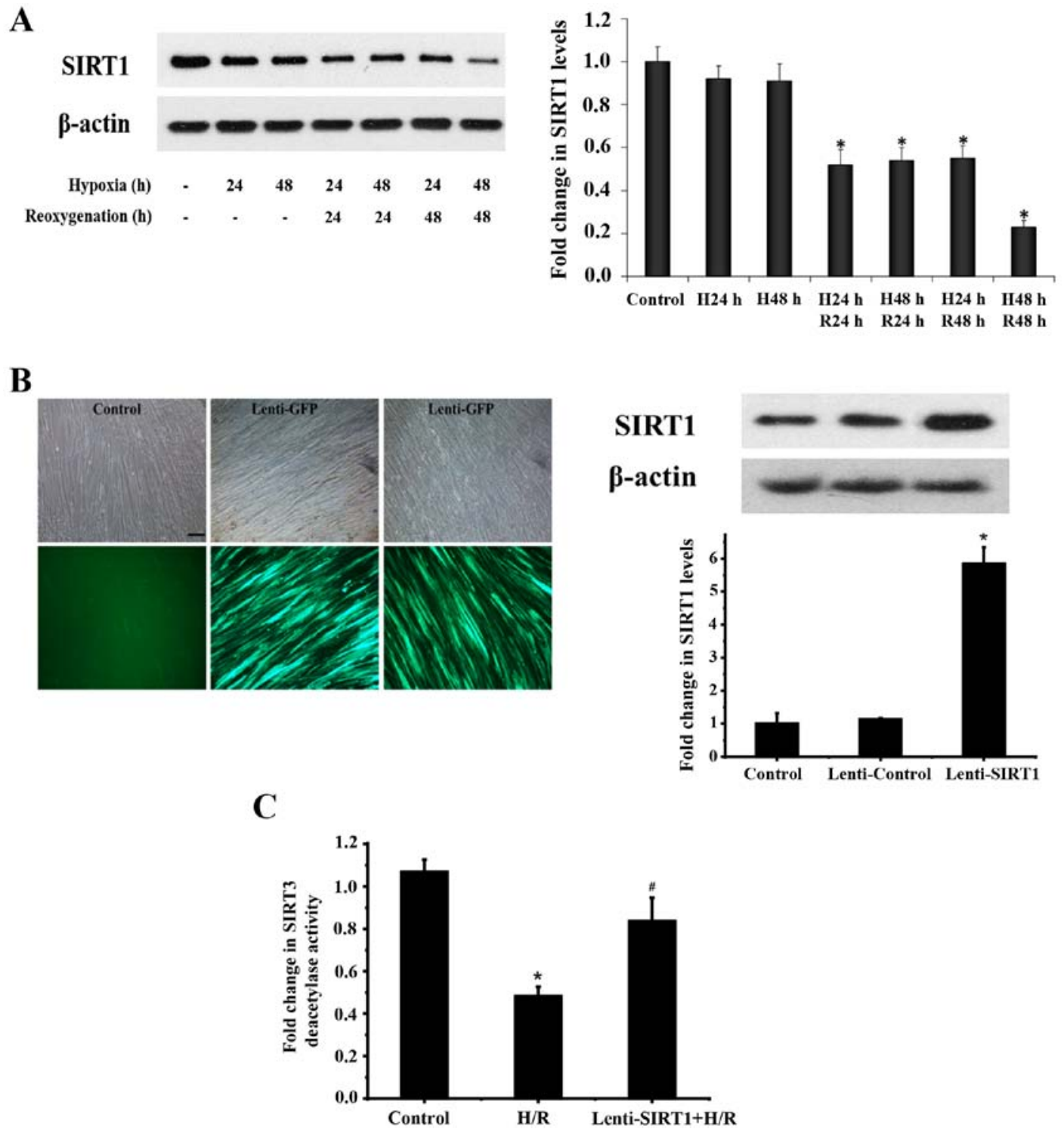

Figure 2. Expression and activation of sirtuin 1 (SIRT1) are reduced by hypoxia/reoxygenation (H/R) in human pulmonary arteriolar smooth muscle cells (PASMCs). (A) Representative immunoblots and densitometric data of 6 separated experiments showed that the expression of SIRT1 was reduced by H/R in human PASMCs. (B) Lentiviral transduction efficiency and GFP expression are shown in human PASMCs. Representative immunoblots and densitometric data showed that lentiviral-expressing SIRT1 induced overexpression of SIRT1 in human PASMCs. (C) Lentiviral-mediated overexpression of SIRT1 restored

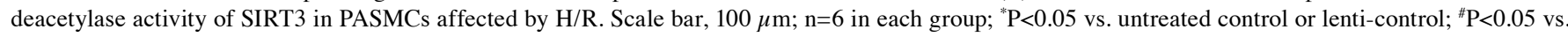
$\mathrm{H} / \mathrm{R}$.

$100 \mu 1$ of $2 \mathrm{X}$ SDS sample buffer. CyPD protein level in the immunoprecipates was analyzed by immunoblotting.

Statistical analysis. Statistical analyses were performed using SPSS version 13.0 (SPSS, Inc., Chicago, IL, USA). All values were presented as mean \pm standard error (SE). One-way or two-way analysis of variance (ANOVA) was used to compare the differences between two groups or among multiple groups, respectively, followed by the post hoc Bonferroni/Dunn test. $\mathrm{P}$-values $<0.05$ were considered statistically significant.

\section{Results}

Expression and activation of SIRTl are reduced by $H / R$ in human PASMCs. We applied antibodies against $\alpha$-SMA, FSP1, and $\mathrm{vWF}$ to determine whether the cultured human PASMCs were contaminated by fibroblasts and endothelial cells. No signals for FSP1 and vWF were detected (Fig. 1), indicating that fibroblasts and endothelial cells were not detected in the isolates. PASMCs were exposed to H/R. As shown in Fig. 2A, the expression of SIRT1 was reduced in the PASMCs during hypoxia for 24-48 $\mathrm{h}$ and reoxygenation for 24-48 $\mathrm{h}$ following hypoxia.

To determine the role of SIRT1 in SIRT3 deacetylase activity during $\mathrm{H} / \mathrm{R}$, human PASMCs were infected with a lentivirus for the overexpression of SIRT1 (Fig. 2B). While SIRT3 deacetylase activity was reduced in cells during $24 \mathrm{~h}$ of hypoxia and $24 \mathrm{~h}$ of reoxygenation, overexpression of SIRT1 restored SIRT3 deacetylase activity reduced by H/R (Fig. 2C).

Expression and release of mitochondrial TFAM are altered by $H / R$ in human PASMCs. To determine PASMC mitochondrial 

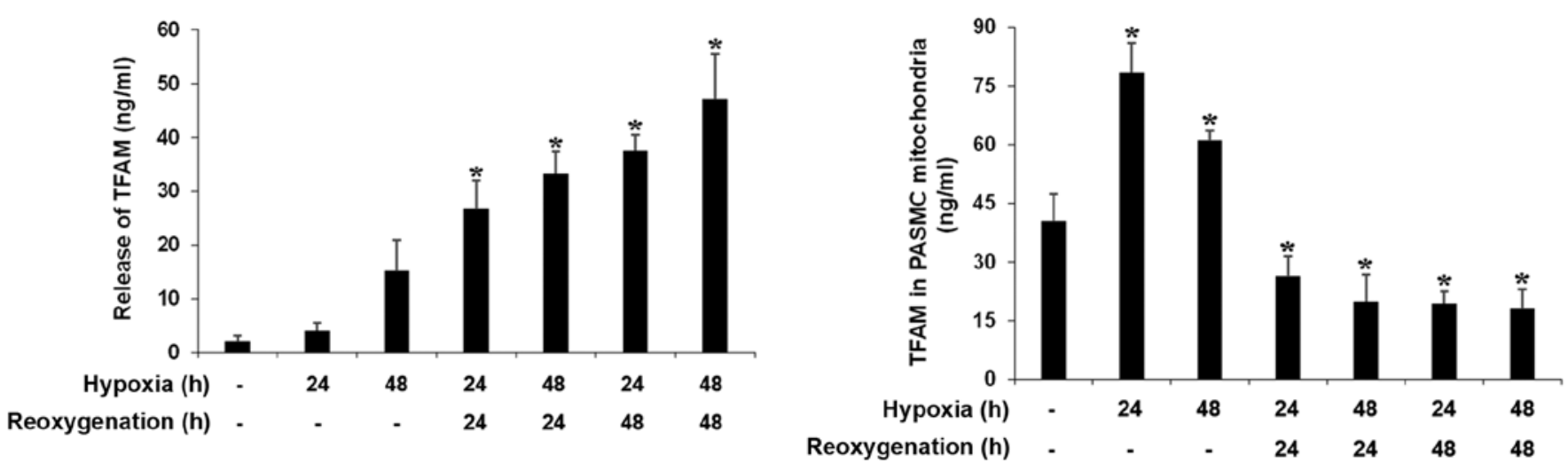

Figure 3. Expression and release of mitochondrial transcription factor A (TFAM) are altered by hypoxia/reoxygenation (H/R) in human pulmonary arteriolar smooth muscle cells (PASMCs). The release of TFAM was increased in a time-dependent manner especially after reoxygenation. TFAM expression in the mitochondria was increased during hypoxia of 24 and $48 \mathrm{~h}$, while the protein expression was decreased during reoxygenation. $\mathrm{n}=5$ in each group; ${ }^{*} \mathrm{P}<0.05$ vs. the untreated control.

biogenesis reprogramming, we detected expression of TFAM in human PASMCs exposed to H/R. As shown in Fig. 3, TFAM levels in the mitochondria were increased at 24 and $48 \mathrm{~h}$ of hypoxia, while TFAM levels in the mitochondria were decreased after reoxygenation. Interestingly, we found the release of TFAM by PASMCs was markedly increased after reoxygenation (Fig. 3).

PGC-1 $\alpha / S I R T 3 / C y P D$ mediates the effect of SIRT1 on TFAM in PASMCs during H/R. To determine how SIRT1 exerts an effect on SIRT3 activity, we knocked down PGC-1 $\alpha$, a downstream target of SIRT1. Knockdown of PGC-1 $\alpha$ abrogated restoration of H/R-induced deacetylase activity of SIRT3 by SIRT1 (Fig. 4A). Overexpression of SIRT1 reduced the acetylation of mitochondrial CyPD, an important mitochondrial member that is involved in regulating the mitochondrial permeability transition, during H/R (Fig. 4B). It has been reported that SIRT3 regulates CyPD deacetylation in cardiac muscle (23). Therefore, we transfected PASMCs with SIRT3 siRNA and found that knockdown of SIRT3 inhibited the effect of SIRT1 on the acetylation of mitochondrial CyPD during H/R (Fig. 4B). These data suggest that SIRT3 deacetylase activity is necessary for SIRT1-regulated acetylation of CyPD during H/R. Importantly, overexpression of SIRT1 increased TFAM levels in mitochondria during H/R (Fig. 4C). Knockdown of SIRT3 or PGC-1 $\alpha$ suppressed the protective effect of SIRT1 on TFAM levels in the mitochondria (Fig. 4C). For release of TFAM by PASMCs, overexpression of SIRT1 decreased the levels of TFAM during H/R. Knockdown of SIRT3 or PGC-1 $\alpha$ suppressed the effect of SIRT1 on the release of TFAM (Fig. 4C).

Stimulation of mitochondrial biogenesis in PASMCs requires functional SIRT1. To determine whether SIRT1 can stimulate mitochondrial biogenesis, we examined the mitochondrial DNA (mtDNA) content in PASMCs. As shown in Fig. 5, mtDNA content was decreased 2.5-fold by H/R. Overexpression of SIRT1 increased mtDNA content. Knockdown of SIRT3 or PGC-1 $\alpha$ suppressed the effect of SIRT1 on mtDNA content. Furthermore, overexpression of SIRT1 increased cellular ATP levels during H/R. Knockdown of SIRT3 or PGC-1 $\alpha$ suppressed the effect of SIRT1 on ATP levels (Fig. 5).

Polydatin improves mitochondrial biogenetic reprogramming and mitochondrial function in PASMCs, but has no effect on SIRT1-knockdown PASMCs. To determine the role of polydatin in mitochondrial biogenesis reprogramming, we treated SIRT1-knockdown PASMCs with polydatin during H/R. As shown in Fig. 6A, polydatin $(50 \mathrm{nM})$ restored SIRT1 levels in human PASMCs exposed to $24 \mathrm{~h}$ reoxygenation after $24 \mathrm{~h}$ hypoxia. Polydatin $(50 \mathrm{nM})$ increased $\mathrm{mtDNA}$ content in the human PASMCs exposed to $24 \mathrm{~h}$ reoxygenation after $24 \mathrm{~h}$ hypoxia. Knockdown of SIRT1 suppressed the effect of polydatin on mtDNA content. We also found that polydatin $(50 \mathrm{nM})$ increased the expression of TFAM in mitochondria during H/R (Fig. 6B). Knockdown of SIRT1 suppressed the effect of polydatin $(50 \mathrm{nM})$ on the expression of TFAM in mitochondria (Fig. 6B). Conversely, polydatin $(50 \mathrm{nM})$ decreased the release of TFAM in the mitochondria during H/R (Fig. 6B). Knockdown of SIRT1 suppressed the effect of polydatin $(50 \mathrm{nM})$ on the release of TFAM (Fig. 6B). Importantly, polydatin $(50 \mathrm{nM})$ increased ATP levels in the PASMCs during H/R (Fig. 6C). Knockdown of SIRT1 suppressed the effect of polydatin $(50 \mathrm{nM})$ on ATP levels (Fig. 6C).

\section{Discussion}

Several in vivo and in vitro studies have demonstrated that histone deacetylase SIRT1 activity is decreased in human lung epithelial cells, macrophages, and lungs of mice exposed to cigarette smoke and in the lungs of patients with chronic obstructive pulmonary disease (COPD) $(24,25)$. Clinical evidence suggests that SIRT1 also affects the clock-dependent metabolome and mitochondrial functions/biogenesis, which are altered during the pathogenesis of airway diseases such as COPD (26). Acute regulation of SIRT1 in response to stressful conditions, such as ischemia and reperfusion are likely to differ according to the type of injury; SIRT1 was found to be downregulated in mice exposed to $20 \mathrm{~min}$ ischemia followed by $24 \mathrm{~h}$ reperfusion (27), but upregulated in hearts subjected to short periods of ischemia and reperfusion (28). We demon- 
$\mathbf{A}$

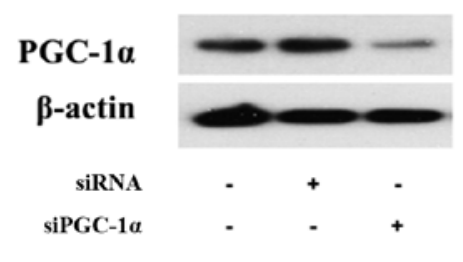

B

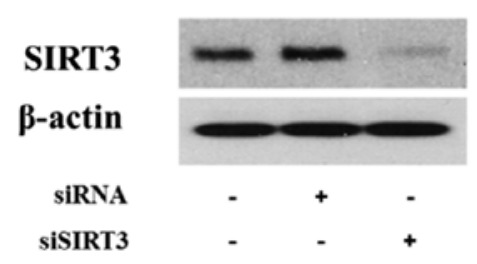

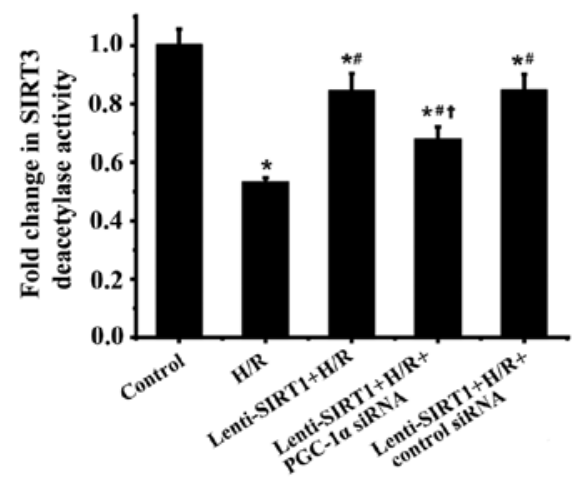

IP:

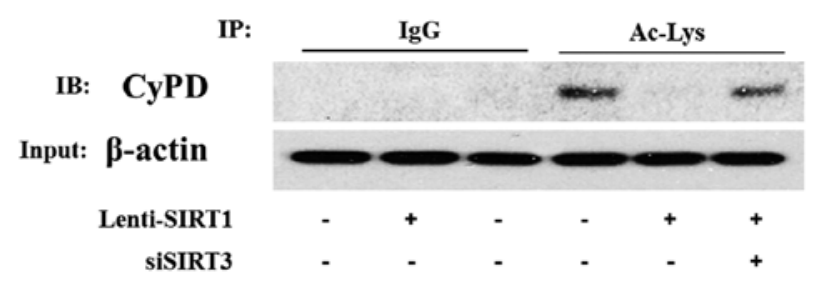

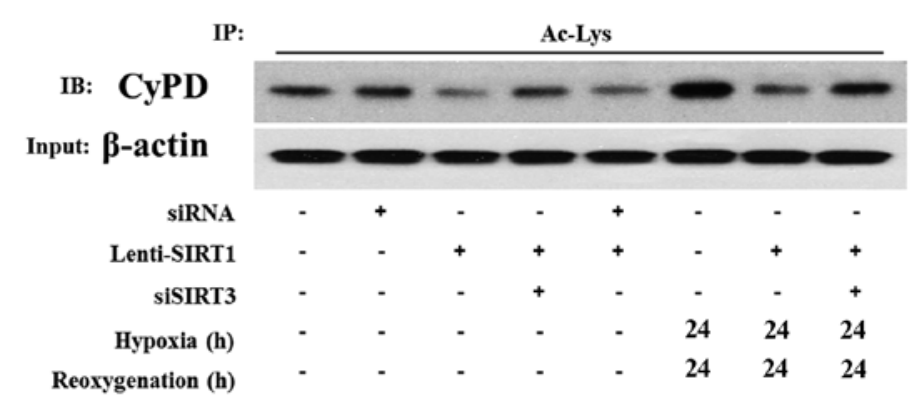

C
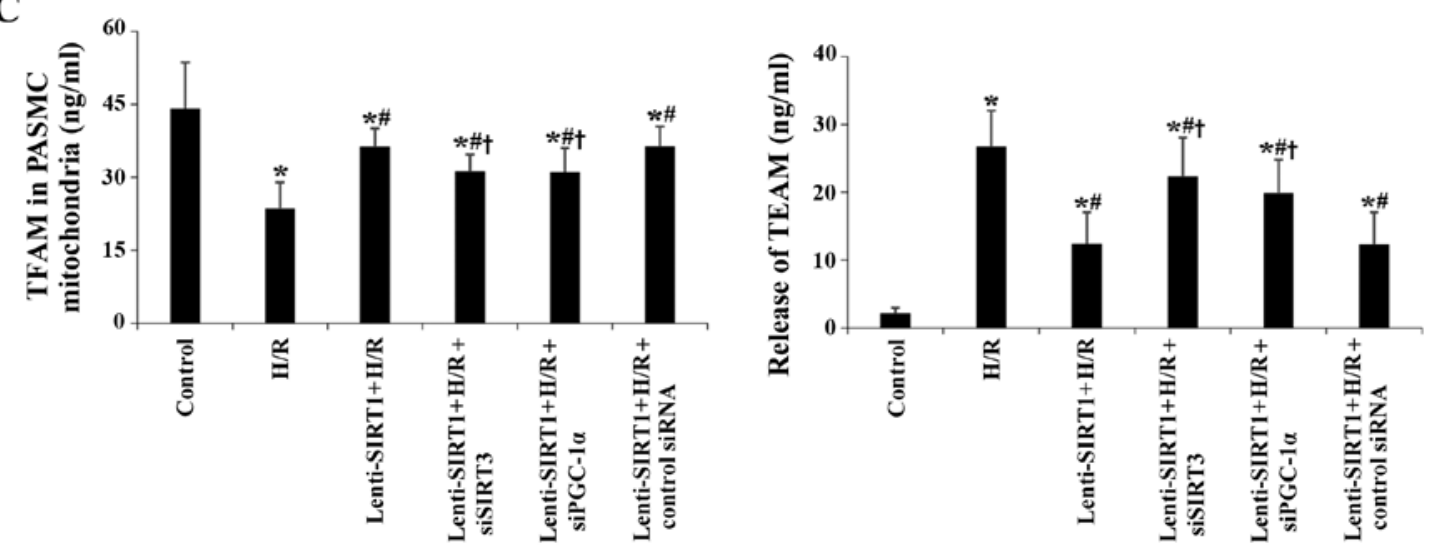

Figure 4. PGC-1 $\alpha /$ SIRT3/cyclophilin D (CyPD) mediates the effect of sirtuin 1 (SIRT1) on TFAM in pulmonary arteriolar smooth muscle cells (PASMCs) during hypoxia/reoxygenation (H/R). (A) Representative immunoblots of 5 separate experiments showed that knockdown of PGC-1 $\alpha$ markedly reduced PGC-1 $\alpha$ protein levels in the PASMCs. Knockdown of PGC-1 $\alpha$ abated restoration by SIRT1 of SIRT3 deacetylase activity induced by H/R. (B) Representative immunoblots of 5 separate experiments showed that knockdown of SIRT3 markedly reduced SIRT3 protein levels in the PASMCs. CoIP assay showed that overexpression of SIRT1 reduced the acetylation of CyPD. Acetylation of CyPD was enhanced by H/R. Knockdown of SIRT3 inhibited the effect of SIRT1 on the acetylation of CyPD in PASMCs exposed to H/R. (C) Overexpression of SIRT1 increased the expression of TFAM in mitochondria during H/R. Conversely, overexpression of SIRT1 decreased the release of TFAM by PASMCs during H/R. Knockdown of PGC-1 $\alpha$ or SIRT3 suppressed the effect of SIRT1 on the expression of TFAM in mitochondria and the release of TFAM. $n=5$ in each group; ${ }^{*} \mathrm{P}<0.05$ vs. untreated control; ${ }^{\prime \prime} \mathrm{P}<0.05$ vs. $\mathrm{H} / \mathrm{R}$; ${ }^{\circ} \mathrm{P}<0.05$ vs. lenti-SIRT1+H/R or lenti-SIRT1+H/R+scrambled siRNA.

strated that human pulmonary arteriolar smooth muscle cells (PASMCs) exposed to $\mathrm{H} / \mathrm{R}$ (mimicking ischemia and reperfusion) have decreased SIRT1 expression compared to control untreated cells. Our previous study showed that SIRT1 positively modulates SIRT3 activity, which plays an important role in protecting mitochondrial function in ASMCs during hemorrhagic shock (6). To investigate the effect of $H / R$ on SIRT1 activity and function, we examined the effect of the overexpression of SIRT1 on SIRT3 deacetylase activity in PASMCs exposed to H/R. Our data showed that $\mathrm{H} / \mathrm{R}$ decreased 
A

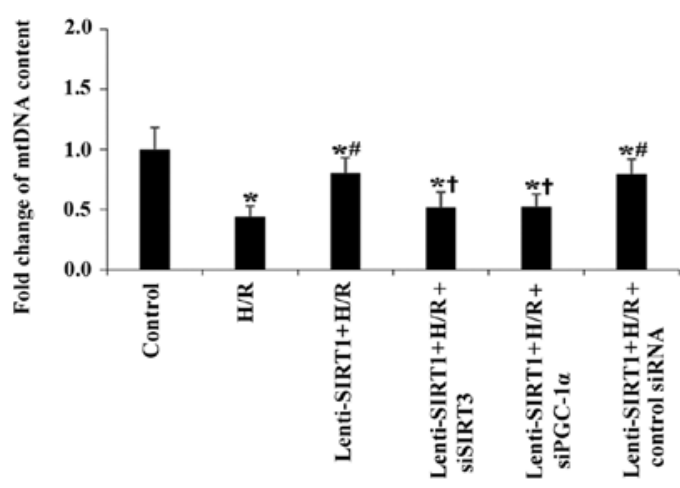

B

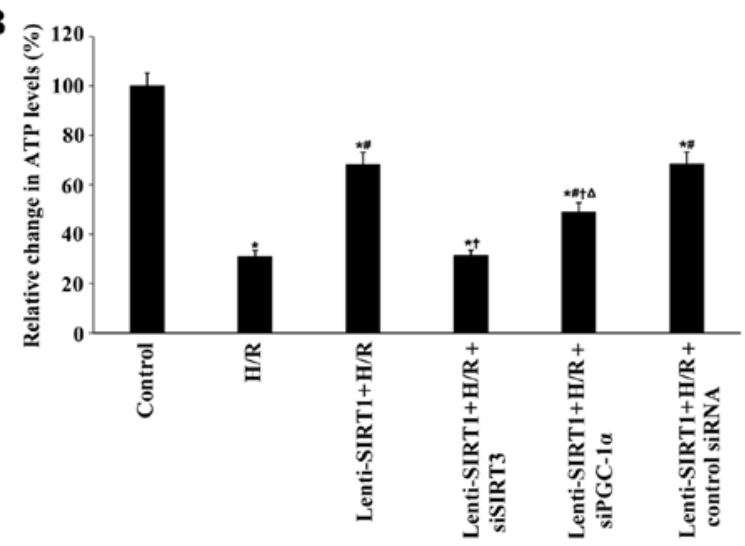

Figure 5. Stimulation of mitochondrial biogenesis in pulmonary arteriolar smooth muscle cells (PASMCs) requires functional sirtuin 1 (SIRT1). (A) Quantitative analysis of the mitochondrial DNA (mtDNA) content relative to nDNA in PASMCs showed that overexpression of SIRT1 increased the expression of TFAM in mitochondria during hypoxia/reoxygenation (H/R). Knockdown of PGC-1 $\alpha$ or SIRT3 suppressed the effect of SIRT1 on mtDNA content. (B) Overexpression of SIRT1 increased ATP levels in PASMCs during H/R. Knockdown of PGC-1 $\alpha$ or SIRT3 suppressed the effect of SIRT1 on ATP levels. $\mathrm{n}=5$ in each group; ${ }^{*} \mathrm{P}<0.05$ vs. untreated control; ${ }^{*} \mathrm{P}<0.05$ vs. $\mathrm{H} / \mathrm{R} ;{ }^{\circ} \mathrm{P}<0.05$ vs. lenti-SIRT1+H/R or lenti-SIRT1+H/R+scrambled siRNA; ${ }^{\wedge} \mathrm{P}<0.05$ vs. lenti-SIRT1+H/R+siSIRT3.

A

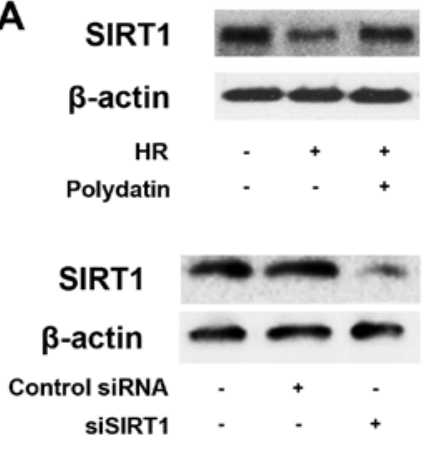

B

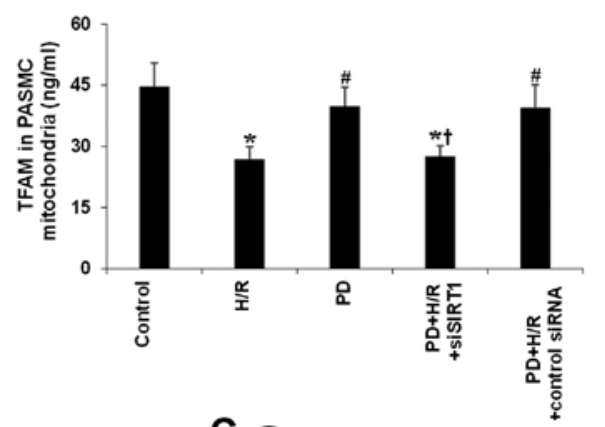

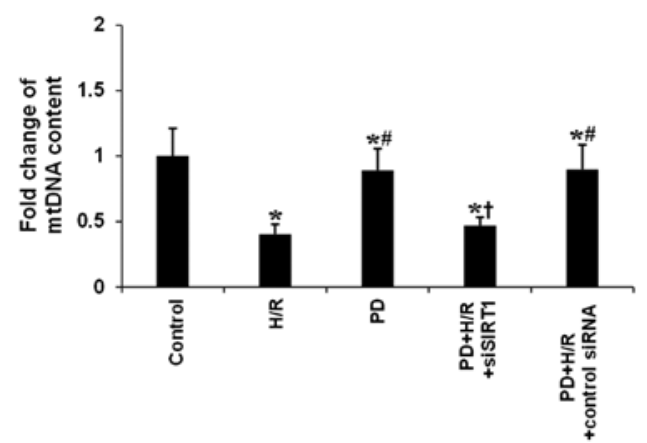

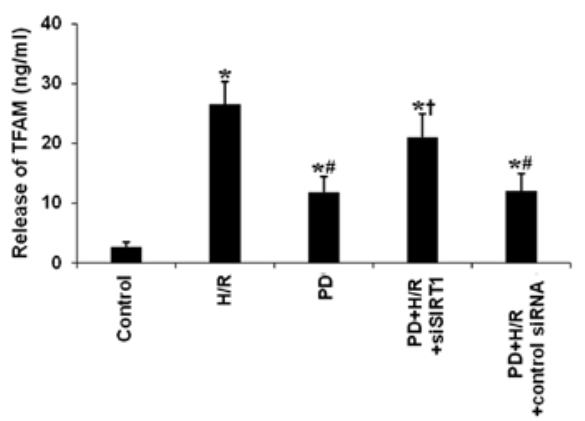

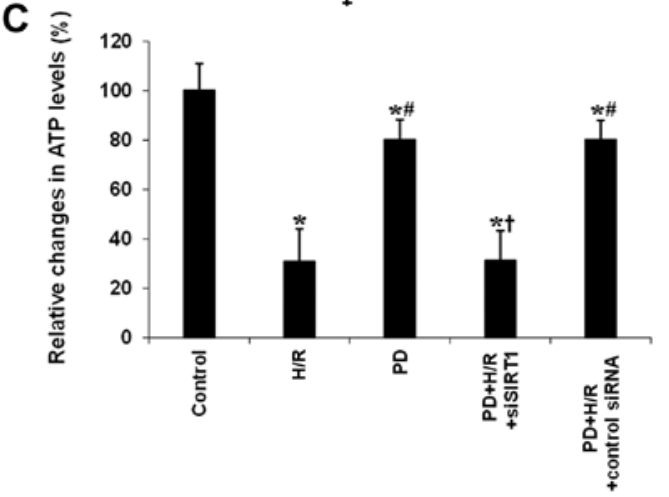

Figure 6. Polydatin improves mitochondrial biogenetic reprogramming and mitochondrial function in pulmonary arteriolar smooth muscle cells (PASMCs), but has no effect on sirtuin 1 (SIRT1)-knockdown PASMCs. (A) Polydatin (PD) (50 nM) restored the expression of SIRT1 at $24 \mathrm{~h}$ reoxygenation after $24 \mathrm{~h}$ hypoxia. SIRT1 siRNA (100 nM) knocked down the expression of SIRT1. Knockdown of SIRT1 suppressed the effect of polydatin on mitochondrial DNA (mtDNA) content during hypoxia/reoxygenation (H/R). (B) Polydatin $(50 \mathrm{nM})$ increased the expression of TFAM in mitochondria during H/R, while polydatin (50 nM) decreased the release of TFAM by PASMCs. Knockdown of SIRT1 suppressed the effect of polydatin $(50 \mathrm{nM})$ on the expression of TFAM in mitochondria and the release of TFAM. (C) Polydatin ( $50 \mathrm{nM}$ ) increased ATP levels in PASMCs during H/R. Knockdown of SIRT1 suppressed the effect of polydatin (50 nM) on ATP levels. $\mathrm{n}=5$ in each group; ${ }^{*} \mathrm{P}<0.05$ vs. untreated control; ${ }^{*} \mathrm{P}<0.05$ vs. H/R; ${ }^{\mathrm{P}} \mathrm{P}<0.05$ vs. lenti-SIRT1+H/R or lenti-control+H/R. H/R, $24 \mathrm{~h}$ reoxygenation after $24 \mathrm{~h}$ hypoxia. 


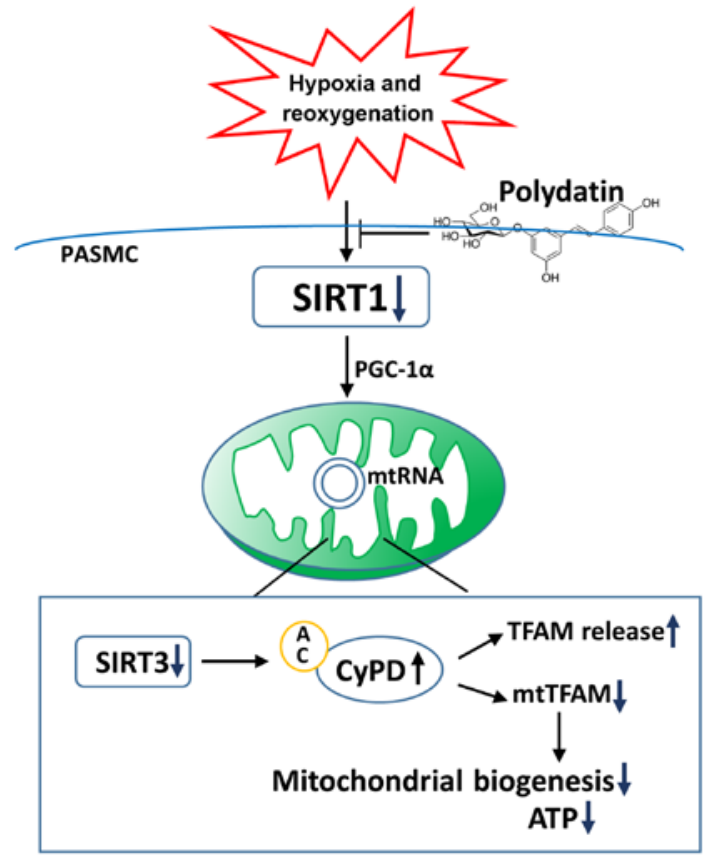

Figure 7. Schematic diagram depicting the mechanism underlying pulmonary arteriolar smooth muscle cell (PASMC) mitochondrial biogenesis reprogramming during hypoxia and reoxygenation. Sirtuin 1 (SIRT1) expression and deacetylation activity are decreased in human pulmonary arteriolar smooth muscle cells (PASMCs) affected by hypoxia and reoxygenation. PGC-1 $\alpha /$ SIRT3/cyclophilin D (CyPD) mediates the protective effect of SIRT1 on expression and release of TFAM and mitochondrial biogenesis and function. Polydatin improves mitochondrial biogenesis and function by enhancing SIRT1 expression in hypoxic human PASMCs.

SIRT3 activity, and overexpression of SIRT1 increased SIRT3 activity in the PASMCs. These results suggest that increased expression of SIRT1 during H/R may protect the mitochondrial biogenesis and function in ASMCs affected by human pulmonary diseases.

Ischemic injury-induced mitochondrial biogenesis reprogramming includes alterations in mtDNA content and TFAM protein expression in the brain and heart $(29,30)$. It was reported that acute ischemic injury significantly increased TFAM protein expression in the retina at 12-72 $\mathrm{h}$ (31). TFAM is rapidly increased in the early neurodegenerative events of neonatal hypoxic-ischemic brain injury (32). Mice lacking TFAM were found to have impaired mtDNA transcription and loss of mtDNA, which led to bioenergetic dysfunction and embryonic lethality (33). In contrast, overexpression of TFAM mediated delayed neuronal death following transient forebrain ischemia in mice (34). Our data showed that TFAM levels were rapidly increased in PASMCs at 24-48 h hypoxia, suggesting these responses may support endogenous repair mechanisms for mtDNA damage following hypoxic-ischemic lung injury. TFAM levels were decreased at 24-48 h reoxygenation, suggesting that mitochondrial function and ultrastructure were damaged. Interestingly, we found that the release of TFAM was increased by PASMCs exposed to H/R. Damage-associated molecular patterns are derived from many sources within the cell, including the plasma membrane, nucleus, cytosol, endoplasmic reticulum and mitochondria. TFAM is a transcription factor for mitochondrial DNA. It is composed of two HMG boxes and a basic carboxyl terminal region (C-tail). Like many other HMG family proteins, TFAM has the ability to bind to DNA in a sequence-independent manner. A growing body of evidence suggests that TFAM may play a crucial role in maintaining mitochondrial DNA as a main component of the nucleoid. Although TFAM is structurally related to HMGB1, it remains unknown whether it can be identified as a DAMP. TFAM can be released into the circulation after hemorrhage to initiate inflammatory responses. Further study is needed to demonstrate the role of TFAM in PASMCs during H/R.

The mechanisms involved in acute regulation of SIRT1 in response to stressful conditions, such as ischemia and reperfusion, are poorly understood. SIRT3 is also emerging as a regulatory protein in the modulation of additional mitochondrial programs. SIRT1 and SIRT3 have been reported to play an important role in mitochondrial morphology and function in ASMCs. However, knowledge of both SIRT1 and SIRT3 in regard to mitochondrial biogenesis is still lacking in PASMCs during H/R. PGC-1 $\alpha$ stimulates mitochondrial biogenesis and promotes the remodeling of muscle tissue to a fiber-type composition that is metabolically more oxidative and less glycolytic in nature (35). SIRT1-promoting mitochondrial biogenesis via PGC- $1 \alpha$ occurs in heart and myotubes $(36,37)$. We found that SIRT1 regulated SIRT3 activity through PGC- $1 \alpha$ in ASMCs exposed to H/R. Inhibition of CyPD-dependent mitochondrial permeability transition pore opening preserved TFAM protein expression and ameliorated mitochondrial dysfunction, leading to protection against hypoxic/ischemic retinal injury (38). We found that knockdown of SIRT3 inhibited the effect of SIRT1 on the acetylation of mitochondrial CyPD under normoxia and during H/R. Knockdown of SIRT3 or PGC-1 $\alpha$ suppressed the inhibitory effects of SIRT1 on the decrease in mitochondrial TFAM expression and increase in the release of TFAM from PASMCs during $\mathrm{H} / \mathrm{R}$. In keeping with its role as a protective mediator in mitochondrial energy production, overexpression of SIRT1 preserved mtDNA content and ATP production in human PASMCs exposed to H/R. Knockdown of SIRT3 or PGC- $1 \alpha$ suppressed the protective effect of SIRT1. Some PGC- $1 \alpha$-independent pathways, such as HIF- $\alpha$, were also found to be involved in SIRT1-mediated mitochondrial regulation in myoblasts and cancer cells $(39,40)$. Further study will be performed to determine the PGC-1 $\alpha$-independent pathways involved in SIRT1-mediated mitochondrial biogenesis and function in PASMCs. Thus, modulation of SIRT1 and its downstream signaling pathways has the potential to protect mitochondrial biogenesis and function in PASMCs during $H / R$.

Data concerning the effects of natural compounds on mitochondrial biogenesis are quite controversial. Several studies indicate that resveratrol and quercetin have some beneficial effects on mitochondrial biogenesis and activity (41). Conversely, other authors have shown that muscle mitochondrial biogenesis should be attributed exclusively to exercise and that quercetin supplementation in the diet had a negligible effect on mitochondria in mice fed with a high-fat diet (42). Oral resveratrol was found to have no effect on mitochondrial biogenesis in skeletal muscle (43). In this study, polydatin, a natural precursor of resveratrol, restored mtDNA and TFAM in mitochondria by enhancing expression of SIRT1 in human PASMCs exposed to H/R. Interestingly, we found that polydatin decreased the release of TFAM through SIRT1 in 
PASMCs exposed to H/R. Our data showed that polydatin had a protective effect on mitochondrial biogenesis and function by enhancing SIRT1 expression.

In conclusion, our results showed that the expression and activity of SIRT1 are decreased in PASMCs exposed to H/R (Fig. 7). PGC-1 $\alpha /$ SIRT3/CyPD mediates the effect of SIRT1 on TFAM expression in mitochondria and the release of TFAM, and mitochondrial biogenesis and function (Fig. 7). Polydatin protects mitochondrial biogenesis and function by enhancing expression of SIRT1 in human PASMCs (Fig. 7). Furthermore, these data indicate the possibility that mitochondrial biogenesis reprogramming may be manipulated as a strategy by which to enhance tolerance against ischemic pulmonary injury.

\section{Acknowledgements}

This study was supported by the Natural Science Foundation of Guangdong Province (no. 2015A030313297) and the National Science Foundation of China (nos. 81200234 and 81600381).

\section{References}

1. Rabe KF, Hurd S, Anzueto A, Barnes PJ, Buist SA, Calverley P, Fukuchi Y, Jenkins C, Rodriguez-Roisin R, van Weel C, et al; Global Initiative for Chronic Obstructive Lung Disease: Global strategy for the diagnosis, management, and prevention of chronic obstructive pulmonary disease: GOLD executive summary. Am J Respir Crit Care Med 176: 532-555, 2007.

2. Gibson PG and Simpson JL: The overlap syndrome of asthma and COPD: What are its features and how important is it? Thorax 64: 728-735, 2009.

3. Evans AM, Hardie DG, Peers C and Mahmoud A: Hypoxic pulmonary vasoconstriction: Mechanisms of oxygen-sensing. Curr Opin Anaesthesiol 24: 13-20, 2011.

4. Freund-Michel V, Khoyrattee N, Savineau JP, Muller B and Guibert C: Mitochondria: Roles in pulmonary hypertension. Int J Biochem Cell Biol 55: 93-97, 2014.

5. Kalogeris T, Baines CP, Krenz M and Korthuis RJ: Cell biology of ischemia/reperfusion injury. Int Rev Cell Mol Biol 298: 229-317, 2012

6. Li P, Meng X, Bian H, Burns N, Zhao KS and Song R: Activation of sirtuin $1 / 3$ improves vascular hyporeactivity in severe hemorrhagic shock by alleviation of mitochondrial damage. Oncotarget 6: 36998-37011, 2015.

7. Powell RD, Swet JH, Kennedy KL, Huynh TT, McKillop IH and Evans SL: Resveratrol attenuates hypoxic injury in a primary hepatocyte model of hemorrhagic shock and resuscitation. J Trauma Acute Care Surg 76: 409-417, 2014.

8. Jian B, Yang S, Chaudry IH and Raju R: Resveratrol restores sirtuin 1 (SIRT1) activity and pyruvate dehydrogenase kinase 1 (PDK1) expression after hemorrhagic injury in a rat model. Mol Med 20: 10-16, 2014.

9. Jian B, Yang S, Chaudry IH and Raju R: Resveratrol improves cardiac contractility following trauma-hemorrhage by modulating Sirt1. Mol Med 18: 209-214, 2012.

10. Waypa GB, Osborne SW, Marks JD, Berkelhamer SK, Kondapalli J and Schumacker PT: Sirtuin 3 deficiency does not augment hypoxia-induced pulmonary hypertension. Am J Respir Cell Mol Biol 49: 885-891, 2013.

11. Paulin R, Dromparis P, Sutendra G, Gurtu V, Zervopoulos S, Bowers L, Haromy A, Webster L, Provencher S, Bonnet S, et al: Sirtuin 3 deficiency is associated with inhibited mitochondrial function and pulmonary arterial hypertension in rodents and humans. Cell Metab 20: 827-839, 2014.

12. Menzies KJ and Hood DA: The role of SirT1 in muscle mitochondrial turnover. Mitochondrion 12: 5-13, 2012.

13. Kong X, Wang R, Xue Y, Liu X, Zhang H, Chen Y, Fang F and Chang Y: Sirtuin 3, a new target of PGC-1alpha, plays an important role in the suppression of ROS and mitochondrial biogenesis. PLoS One 5: e11707, 2010.
14. Baines CP, Kaiser RA, Purcell NH, Blair NS, Osinska H, Hambleton MA, Brunskill EW, Sayen MR, Gottlieb RA, Dorn GW, et al: Loss of cyclophilin D reveals a critical role for mitochondrial permeability transition in cell death. Nature 434: 658-662, 2005

15. Marzetti E, Calvani R, Cesari M, Buford TW, Lorenzi M, Behnke BJ and Leeuwenburgh C: Mitochondrial dysfunction and sarcopenia of aging: From signaling pathways to clinical trials. Int J Biochem Cell Biol 45: 2288-2301, 2013.

16. Civitarese AE, Carling S, Heilbronn LK, Hulver MH, Ukropcova B, Deutsch WA, Smith SR and Ravussin E; CALERIE Pennington Team: Calorie restriction increases muscle mitochondrial biogenesis in healthy humans. PLoS Med 4: e76, 2007.

17. Chaung WW, Wu R, Ji Y, Dong W and Wang P: Mitochondrial transcription factor A is a proinflammatory mediator in hemorrhagic shock. Int J Mol Med 30: 199-203, 2012.

18. Liu HB, Meng QH, Huang C, Wang JB and Liu XW: Nephroprotective effects of polydatin against ischemia/reperfusion injury: A role for the PI3K/Akt signal pathway. Oxid Med Cell Longev 2015: 362158, 2015.

19. Li XH, Gong X, Zhang L, Jiang R, Li HZ, Wu MJ and Wan JY: Protective effects of polydatin on septic lung injury in mice via upregulation of HO-1. Mediators Inflamm 2013: 354087, 2013.

20. Zeng Z, Yang Y, Dai X, Xu S, Li T, Zhang Q, Zhao KS and Chen Z: Polydatin ameliorates injury to the small intestine induced by hemorrhagic shock via SIRT3 activation-mediated mitochondrial protection. Expert Opin Ther Targets 20: 645-652, 2016.

21. Wang X, Song R, Chen Y, Zhao M and Zhao KS: Polydatin - a new mitochondria protector for acute severe hemorrhagic shock treatment. Expert Opin Investig Drugs 22: 169-179, 2013.

22. Li P, Wang X, Zhao M, Song R and Zhao KS: Polydatin protects hepatocytes against mitochondrial injury in acute severe hemorrhagic shock via SIRT1-SOD2 pathway. Expert Opin Ther Targets 19: 997-1010, 2015.

23. Hafner AV, Dai J, Gomes AP, Xiao CY, Palmeira CM, Rosenzweig A and Sinclair DA: Regulation of the mPTP by SIRT3-mediated deacetylation of CypD at lysine 166 suppresses age-related cardiac hypertrophy. Aging (Albany NY) 2: 914-923, 2010.

24. Becker RH, Sha S, Frick AD and Fountaine RJ: The effect of smoking cessation and subsequent resumption on absorption of inhaled insulin. Diabetes Care 29: 277-282, 2006.

25. Rajendrasozhan S, Yang SR, Kinnula VL and Rahman I: SIRT1, an antiinflammatory and antiaging protein, is decreased in lungs of patients with chronic obstructive pulmonary disease. Am J Respir Crit Care Med 177: 861-870, 2008.

26. Yao H, Sundar IK, Huang Y, Gerloff J, Sellix MT, Sime PJ and Rahman I: Disruption of sirtuin 1-mediated control of circadian molecular clock and inflammation in chronic obstructive pulmonary disease. Am J Respir Cell Mol Biol 53: 782-792, 2015.

27. Hsu CP, Zhai P, Yamamoto T, Maejima Y, Matsushima S, Hariharan N, Shao D, Takagi H, Oka S and Sadoshima J: Silent information regulator 1 protects the heart from ischemia/reperfusion. Circulation 122: 2170-2182, 2010.

28. Cattelan A, Ceolotto G, Bova S, Albiero M, Kuppusamy M, De Martin S, Semplicini A, Fadini GP, de Kreutzenberg SV and Avogaro A: NAD(+)-dependent SIRT1 deactivation has a key role on ischemia-reperfusion-induced apoptosis. Vascul Pharmacol 70: 35-44, 2015.

29. Uittenbogaard $\mathrm{M}$ and Chiaramello A: Mitochondrial biogenesis: a therapeutic target for neurodevelopmental disorders and neurodegenerative diseases. Curr Pharm Des 20: 5574-5593, 2014.

30. Pisano A, Cerbelli B, Perli E, Pelullo M, Bargelli V, Preziuso C, Mancini M, He L, Bates MG, Lucena JR, et al: Impaired mitochondrial biogenesis is a common feature to myocardial hypertrophy and end-stage ischemic heart failure. Cardiovasc Pathol 25: 103-112, 2016.

31. Lee D, Kim KY, Noh YH, Chai S, Lindsey JD, Ellisman MH, Weinreb RN and Ju WK: Brimonidine blocks glutamate excitotoxicity-induced oxidative stress and preserves mitochondrial transcription factor a in ischemic retinal injury. PLoS One 7: e47098, 2012.

32. Yin W, Signore AP, Iwai M, Cao G, Gao Y and Chen J: Rapidly increased neuronal mitochondrial biogenesis after hypoxic-ischemic brain injury. Stroke 39: 3057-3063, 2008.

33. Larsson NG, Wang J, Wilhelmsson H, Oldfors A, Rustin P, Lewandoski M, Barsh GS and Clayton DA: Mitochondrial transcription factor $\mathrm{A}$ is necessary for mtDNA maintenance and embryogenesis in mice. Nat Genet 18: 231-236, 1998. 
34. Hokari M, Kuroda S, Kinugawa S, Ide T, Tsutsui H and Iwasaki Y: Overexpression of mitochondrial transcription factor A (TFAM) ameliorates delayed neuronal death due to transient forebrain ischemia in mice. Neuropathology 30: 401-407, 2010.

35. Liang $\mathrm{H}$ and Ward WF: PGC-1alpha: A key regulator of energy metabolism. Adv Physiol Educ 30: 145-151, 2006.

36. Lehman JJ, Barger PM, Kovacs A, Saffitz JE, Medeiros DM and Kelly DP: Peroxisome proliferator-activated receptor gamma coactivator-1 promotes cardiac mitochondrial biogenesis. J Clin Invest 106: 847-856, 2000.

37. Wu Z, Puigserver P, Andersson U, Zhang C, Adelmant G, Mootha V, Troy A, Cinti S, Lowell B, Scarpulla RC, et al: Mechanisms controlling mitochondrial biogenesis and respiration through the thermogenic coactivator PGC-1. Cell 98: 115-124, 1999.

38. Kim SY, Shim MS, Kim KY, Weinreb RN, Wheeler LA and Ju WK: Inhibition of cyclophilin D by cyclosporin A promotes retinal ganglion cell survival by preventing mitochondrial alteration in ischemic injury. Cell Death Dis 5: e1105, 2014.

39. Gomes AP, Price NL, Ling AJ, Moslehi JJ, Montgomery MK Rajman L, White JP, Teodoro JS, Wrann CD, Hubbard BP, et al: Declining $\mathrm{NAD}(+)$ induces a pseudohypoxic state disrupting nuclear-mitochondrial communication during aging. Cell 155 1624-1638, 2013.
40. Joo HY, Yun M, Jeong J, Park ER, Shin HJ, Woo SR, Jung JK, Kim YM, Park JJ, Kim J, et al: SIRT1 deacetylates and stabilizes hypoxia-inducible factor- $1 \alpha(\mathrm{HIF}-1 \alpha)$ via direct interactions during hypoxia. Biochem Biophys Res Commun 462: 294-300, 2015.

41. Lagouge M, Argmann C, Gerhart-Hines Z, Meziane H, Lerin C, Daussin F, Messadeq N, Milne J, Lambert P, Elliott P, et al: Resveratrol improves mitochondrial function and protects against metabolic disease by activating SIRT1 and PGC-1alpha. Cell 127: 1109-1122, 2006.

42. Kwon SM, Park HG, Jun JK and Lee WL: Exercise, but not quercetin, ameliorates inflammation, mitochondrial biogenesis, and lipid metabolism in skeletal muscle after strenuous exercise by high-fat diet mice. J Exerc Nutrition Biochem 18: 51-60, 2014. 43. Higashida K, Kim SH, Jung SR, Asaka M, Holloszy JO and Han DH: Effects of resveratrol and SIRT1 on PGC-1 $\alpha$ activity and mitochondrial biogenesis: A reevaluation. PLoS Biol 11: e1001603, 2013. 